\title{
Intra- and interfractional variations in geometric arrangement between lung tumours and implanted markers.
}

\author{
AUTHOR(S): \\ Ueki, Nami; Matsuo, Yukinori; Nakamura, Mitsuhiro; \\ Mukumoto, Nobutaka; lizuka, Yusuke; Miyabe, Yuki; \\ Sawada, Akira; Mizowaki, Takashi; Kokubo, Masaki; Hiraoka, \\ Masahiro
}

\section{CITATION:}

Ueki, Nami ... [et al]. Intra- and interfractional variations in geometric arrangement between lung tumours and implanted markers.. Radiotherapy and oncology: journal of the European Society for Therapeutic Radiology and Oncology 2014, 110(3): 523-528

\section{ISSUE DATE:}

2014-03

URL:

http://hdl.handle.net/2433/187802

\section{RIGHT:}

@ 2014 Elsevier Ireland Ltd.; この論文は出版社版でありません。引用の 際には出版社版をご確認ご利用ください。; This is not the published version. Please cite only the published version. 
$1 / 23$

1 Title

2 Intra- and Interfractional Variations in Geometric Arrangement between Lung Tumours and 3 Implanted Markers

4

5

6

9 Mizowaki, M.D., Ph.D., ${ }^{1}$ Masaki Kokubo, M.D., Ph.D, ${ }^{3}$ and Masahiro Hiraoka, M.D., Ph.D. ${ }^{1}$

10

11

12

13

14 School of Medicine, Kyoto, Japan.

$15{ }^{2}$ Faculty of medical science, Kyoto College of Medical Science, Nantan, Japan.

$16{ }^{3}$ Department of Radiation Oncology, Kobe City Medical Center General Hospital, Kobe, 17 Japan

18

19

20

21 Yukinori Matsuo, M.D., Ph.D., Department of Radiation Oncology and Image-applied

22 Therapy, Graduate School of Medicine, Kyoto University,

23 Phone: +81-75-751-3762, fax: +81-75-771-9749

24 E-mail: ymatsuo@kuhp.kyoto-u.ac.jp 
28 Running header

29 Variation between lung tumour and markers

30

$31 \quad$ Keywords

32 Lung cancer, fiducial marker, positional variation, 4DCT.

33

$34 \quad$ Number of pages: 17

35 Number of tables and figures: 5 
$3 / 23$

\section{Abstract}

Purpose: To quantify the intra- and interfractional variations between lung tumours and implanted markers.

Materials and Methods: Gold markers were implanted transbronchially around a lung tumour in fifteen patients. They underwent four-dimensional computed tomography scans twice, and the centroids of the tumour and markers were determined. Intrafractional variations were defined as the residual tumour motions relative to the markers due to respiration from the end-exhale phase. Interfractional variations were defined as the residual setup errors after correction for the position of the implanted markers in end-exhale phase images.

Results: The intrafractional variations differed between patients. The root mean squares of standard deviations for each phase were $0.6,0.9$, and $1.5 \mathrm{~mm}$ in the right-left, anterior-posterior, and superior-inferior directions, respectively. The maximum difference in intrafractional variation among 10 phases was correlated with the amplitude of tumour motion in all directions and the tumour-marker distance in the anterior-posterior and superior-inferior directions. The interfractional variations were within $2.5 \mathrm{~mm}$.

Conclusions: The intrafractional variations differed according to the amount of tumour motion and the tumour-marker distance. Additionally, interfractional variations of up to 2.5 mm were observed. Thus, a corresponding margin should be considered during implanted marker-based beam delivery to account for these variations. 
$4 / 23$ irradiation treatment.

\section{Introduction} is known.

Stereotactic body radiation therapy (SBRT) is an innovative technique that delivers high-dose radiation limited precisely to the region of the tumour $[1,2]$. In SBRT for targets affected by respiratory motion, such as lung tumours, appropriate motion management is recommended to reduce doses delivered to the surrounding normal tissues. Several methods of accounting for respiratory motion have been developed, including methods in which the radiation delivery is synchronised with respiration; i.e. the dynamic tumour tracking (DTT) method and the respiratory gating method [3].

With the above respiratory-synchronised methods, markers implanted either in the tumour itself or nearby are often used as the internal surrogate to localise the tumour position [4-6]. However, the position of the implanted markers does not always represent the tumour position because the tumour and markers move non-synchronously during respiration, especially in cases in which the markers were located slightly distal from the tumour [7]. This intrafractional positional difference between the tumour and markers should be incorporated into the DTT or respiratory gating irradiation treatment plan by using a wider gating window, within which the beam is delivered during the respiration cycle. Furthermore, the relative position of the tumour with respect to the markers may vary from day to day; therefore, the interfractional positional difference must be addressed. However, little about these variations

The purpose of this study was to quantify the intra- and interfractional variations between the lung tumour position and the position of the implanted markers to evaluate the margin necessary to account for the associated errors during respiratory-synchronised 
$5 / 23$

79

80

81

82

\section{Materials and Methods}

\section{Patients and implanted markers}

Fifteen patients who underwent SBRT for a solitary lung tumour were enrolled in this study. With the approval of the Institutional Review Board, written informed consent was obtained from all patients. One to two weeks prior to the date of the computed tomography (CT) simulation, four or five disposable gold markers (Olympus Corporation, Tokyo, Japan), spherical markers with a diameter of $1.5 \mathrm{~mm}$, were implanted transbronchially. The insertion technique was similar to the one reported by Harada et al [4]. Prior to the implantation, the relative position between tumour and bronchi was evaluated on the multiplanar reformatted CT images. The markers were implanted into the peripheral surrounding bronchi near tumour under fluoroscopy guidance. A total of 66 markers were placed. The median interval between marker placement and the CT simulation was 8 (range, 2 to 16) days. Twelve markers were coughed up before CT simulation. After CT simulation, 2 markers were coughed up on the seventh and thirteenth day, and 1 marker migrated on the sixth day after insertion. The markers that coughed up or migrated after CT simulation during the treatment period were excluded from this analysis. No adverse effect associated with the implantation was observed. The characteristics of patients and tumours are shown in Table 1.

\section{Patient set-up and four-dimensional CT data acquisition}

The patients were immobilised using vacuum immobilisation devices: BodyFix system (Elekta AB, Stockholm, Sweden) or Esform (Engineering System, Nagano, Japan). After set-up with skin marks, four-dimensional CT (4DCT) data were acquired using a 16-multidetector row CT: LightSpeed RT or BrightSpeed (General Electric Healthcare, Waukesha, WI, USA) with an axial slice thickness of $2.5 \mathrm{~mm}$. The cine duration time of the scan at each couch position was set to 6.0 or $7.0 \mathrm{~s}$, which was more than the maximum 
$6 / 23$

104 observed respiratory period. Simultaneously, the respiratory phase was monitored using the

105 Varian Real-time Position Management system (Varian Medical Systems, Palo Alto, CA,

106 USA) under free breathing without coaching. CT slices and respiratory phase data were

107 transferred to the Advantage SIM workstation (General Electric Healthcare, Waukesha, WI,

108 USA) and sorted into 10 respiratory phase bins. Motion phases were assigned for each

109 respiratory phase as percentages; end-inhalation corresponded to $0 \%$ and end-exhalation to

$11050 \%$. 4DCT scans were performed during the CT simulation (CT-1) and repeated once during

111 the course of treatment (CT-2). Fifteen pairs, corresponding to a total of thirty 4DCT scans,

112 were obtained. The median period from the day of CT-1 until the day of CT-2 was 8 days

113 (range, 4 to 12). All 4DCT datasets were imported into a commercial radiotherapy planning system, iPlan 4.5.1 (BrainLAB AG, Fieldkirchen, Germany).

117 The intrafractional variations assessed in this study were defined as the residual tumour

118 motions relative to the markers due to respiration. In all 10 phases of the CT-1 scans, gross tumours and implanted markers were contoured manually with a pulmonary window setting

120 (window level, -700 Hounsfield units; window width, 2000 Hounsfield units) by a single

121 radiation oncologist. The centroid of the tumour $\mathrm{G}_{t, n}=\left(x_{t, n}, y_{t, n}, z_{t, n}\right)$ and the centroids of all 3

122 to 5 markers $\mathrm{G}_{m, n}=\left(x_{m, n}, y_{m, n}, z_{m, n}\right)$ were recorded at $n \%$ respiratory phase $(0 \leq n \leq 90)$. The

123 coordinates $(x, y, z)$ correspond to the right-left (RL), anterior-posterior (AP), and 124 superior-inferior (SI) directions, respectively. Along each axis, a positive value corresponds to

125 the right, anterior, and superior directions. The relative position of the tumour and centroid of 126 markers for each phase was represented by the vector $\mathrm{V}_{n}=\mathrm{G}_{t, n}-\mathrm{G}_{m, n}$. Using the relative 127 positions on $50 \%$ phase images $\left(\mathrm{V}_{50}\right)$ as a reference, the error $\mathrm{E}_{n}=\mathrm{V}_{n}-\mathrm{V}_{50}$ was calculated. 128 The mean $\left(\mathrm{M}_{n}\right)$ and standard deviation $\left(\mathrm{SD}_{n}\right)$ of $\mathrm{E}_{n}$ in fifteen 4DCT CT-1 datasets were also 
calculated. The mean and SD of $\mathrm{M}_{n}(0 \leq n \leq 90)$ were calculated to evaluate systematic

130 displacement between respiratory phases. The root mean square (RMS) of $\mathrm{SD}_{n}(0 \leq n \leq 90)$ was

131 calculated to evaluate interpatient variations. The range of intrafractional variations is defined

132 as the maximum difference in $\mathrm{E}_{n}$ among 10 phases for each direction. To evaluate the

133 influence of the tumour motion amplitude and the tumour-marker distance on the ranges of

134 intrafractional variations, a multiple linear regression analysis was performed. The tumour

135 motion amplitude was defined as the maximal difference in the tumour centroid position

136 among the 10 respiratory phases in each direction. The tumour-marker distance in each

137 direction was defined as the distance between the tumour centroid and the centroid of all 3 to

1385 markers in the $50 \%$ phase images.

139 The interfractional variations in this study represent the residual setup errors after

140 correction based on the implanted markers. Firstly, to correct the rotational set up errors, the

$14150 \%$ phase images for CT-2 were rigidly registered to the $50 \%$ phase images of CT- 1 based on

142 bony structure. Then the translational errors were modified by registering those images based

143 on the marker centroids. The interfractional variations were evaluated as the residual

144 difference in the tumour centroids for each direction between CT-1 and CT-2 for each patient.

\section{Results}

147 Tumour motion amplitude and tumour-marker distance

148 The median (range) tumour motion amplitudes in CT-1 were $1.8 \mathrm{~mm}$ (0.4 to 5.6), $3.1 \mathrm{~mm}(0.6$

149 to 7.8 ), and $8.2 \mathrm{~mm}$ (0.9 to 28.9 ) in the RL, AP, and SI directions, respectively. The median

150 values (range) of the distance between the tumour centroid and the centroid of all markers in

151 the $50 \%$ phase images for CT- 1 were $11.9 \mathrm{~mm}$ (1.9 to 30.5$), 8.1 \mathrm{~mm}(0.7$ to 35.3$)$, and 10.3

$152 \mathrm{~mm}(0.3$ to 30.1$)$ in the RL, AP, and SI directions, respectively. 
$8 / 23$

154

155

156

157

158

159

160

161

162

163

164

165

166

167

168

169

170

171

172

173

174

175

176

177

178

\section{Intrafractional variations}

The divergence in the range of intrafractional variations between patients is shown in Fig. 1, and the values of $\mathrm{E}_{n}$ in the respiratory phases $(0 \leq n \leq 90)$ are shown in Fig. 2 . The means $\pm \mathrm{SD}$ of $\mathrm{M}_{n}(0 \leq n \leq 90)$ were $0.1 \pm 0.1 \mathrm{~mm}, 0.3 \pm 0.2 \mathrm{~mm}$, and $0.0 \pm 0.2 \mathrm{~mm}$, and the RMS of $\mathrm{SD}_{n}$ were $0.6 \mathrm{~mm}, 0.9 \mathrm{~mm}$, and $1.5 \mathrm{~mm}$ in the RL, AP, and SI directions, respectively. These results indicate that the systematic difference between respiratory phases is negligible. In addition, as shown in Fig. 2, the further towards inhale then the greater the intrafractional variations.

The tumour motion amplitude was positively correlated with the range of intrafractional variations in all directions, and the tumour-marker distances were also positively correlated in the AP and SI directions (Table 2).

\section{Interfractional variations}

The median (range) interfractional variations were $-0.1 \mathrm{~mm}$ ( -2.4 to 0.7$), 0.1 \mathrm{~mm}(-2.3$ to 2.4$)$, and $-0.6 \mathrm{~mm}$ (-1.3 to 1.6$)$ in the RL, AP, and SI directions, respectively. As shown in Fig. 3, all interfractional variations were within $2.5 \mathrm{~mm}$; the greatest variations were in the AP direction. The $95^{\text {th }}$ percentiles of interfractional variations for one side of each direction were 0.6 and $2.1 \mathrm{~mm}$ to the right and left, 1.9 and $2.1 \mathrm{~mm}$ in the anterior and posterior directions, and 1.6 and $1.3 \mathrm{~mm}$ in the superior and inferior directions.

\section{Discussion}

Implanted markers are often used as a surrogate for the tumour position in radiation therapy for lung tumours. The transcutaneous and transbronchial approaches are the two major methods for implantation of markers in the vicinity of lung tumours [8-13]. These procedures may cause pneumothorax as a complication, which can delay radiation delivery and could be life-threatening for those with comorbidities. The incidences of all pneumothorax and of those 
179

180

181

182

requiring chest tube placement after transcutaneous implantation have been reported to be 30 to $67 \%$ and 16 to $40 \%$, respectively [8-10]. By contrast, the reported incidence of pneumothorax with the transbronchial approach is low [10-13] and in our series no complication was observed. Therefore, the transbronchial approach is preferable due to its less invasive nature. However, the placement of markers near or inside the tumour is more difficult with the transbronchial approach than with the transcutaneous approach, because in the former the markers are placed along the small bronchi near a tumour. The greater distance between the tumour and markers leads to a larger positional error [14]. This error must be considered when performing radiotherapy using markers placed outside the tumour. In the current study, we quantified the intra- and interfractional positional variations between the lung tumour and implanted markers using 4DCT scans to determine the necessary margin for respiratory-synchronised irradiation using implanted markers. Another issue about the markers implanted transbronchially is the low fixation rate. In our series, the fixation rate of implanted markers was 77.3\%: 51 of 66 markers implanted markers were fixed throughout treatment. This is comparable to the reported fixation rate using the same insertion technique [11]. Due to the low fixation rate, we inserted 4 or 5 markers to avoid an additional insertion procedure and used multiple markers as a surrogate for the tumour position to address the change in geometric arrangement of markers by dislocation.

Although several authors reported the intrafractional verification of the tumour position by the kilo-voltage $(\mathrm{kV})$ X-ray images during gating irradiation, they calculated the tumour positions from the detected positions of the implanted markers assuming the relative position between the tumour and markers were constant $[7,15]$. The planar $\mathrm{kV}$ X-ray imaging is superior to CT in terms of the temporal resolution but it is difficult to quantify the motion of tumor itself accurately on the projected images. To quantify the variations between the tumour and implanted markers, we used 4DCT. Two studies are available which evaluated the 
$10 / 23$

204 geometrical difference between tumour and markers due to respiration using 4DCT. Smith et 205 al. analysed the motion of lung tissue in 10 patients with deformable registration between 206 exhalation and inhalation of 4DCT scans and reported stronger correlations between tumour 207 and surrounding lung tissues in the upper lobes than in the lower lobes [16]. Finally, they 208 concluded that the correlation between the tumour and the surrounding tissue was highly specific to the patient and lobe [16]. Since the amplitude of the tumour motion is typically smaller in the upper lobe than that in the lower lobe, then it is likely the bigger variations observed in lower lobe tumours by Smith et al. may be related to the amplitude of motion.

212 Yamazaki et al. evaluated the distances between tumours and the distal bronchi during 213 respiration cycle with 4DCT for 8 patients. They showed that the distances in the mid-inhale 214 to end-inhale phase images were significantly larger than the distances in the end-exhale 215 phase images [17]. Smith et al. and Yamazaki et al. suggested that markers that are closer to 216 the tumour give a more accurate representation of tumour motion [16,17]. These results are 217 consistent with our findings: the values needed to compensate for the intrafractional variations 218 differed between patients, and depended on the amplitude of tumour motion and the tumour-marker distance.

Moreover, our results indicated that the intrafractional errors were different for each patient both in direction and in amplitude, as shown in Fig 1. A uniform isotropic margin was not adequate to cover the errors observed. Consequently, the variations must be evaluated on a per-patient basis and compensated for by addition of a patient-specific margin in DTT or gating irradiation treatment with a wider gating window. In our treatment planning process of DTT, we create an enlarged target volume which cover the intrafractional variations in the following steps. Firstly, 4DCT images from each phase are translated based on the centroid of 227 the markers. Then, the phase images are superimposed onto the $50 \%$ phase image that is used 
$11 / 23$

229

tumour volumes on all fused phase image. This enlarged volume can compensate for the patient-specific intrafractional variations.

All interfractional variations in the present study were within $2.5 \mathrm{~mm}$. Previous reports on interfractional variations between lung tumours and implanted markers are summarised in Supplementary Table 1 (Electronic Appendix). The reported values are larger than those in this study. This discrepancy may be attributed to two causes. One is a change in tumour-marker distance during the course of treatment. Several investigators reported tumour shrinkage and deformation after radiotherapy with conventional fractionation [5,10,14], which altered the distance. Meanwhile, Imura et al. [11] evaluated the interfractional variations in the distances between markers using orthogonal X-ray images with a median treatment time of 6 days, and showed that the variations during treatment were within $2 \mathrm{~mm}$ in $95 \%$ of cases. Their results support our finding that the interfractional variation between the tumour and markers was smaller in those undergoing hypofractionated treatment than in those undergoing conventional fractionation. A second interesting finding was the respiratory phase reproducibility. Van der Voort van Zyp et al. assessed marker displacement compared to the centroid of the tumour in patients that underwent SBRT; however, their results were influenced by the nonsynchronous tumour-marker motion due to divergence in the timing of breath holding [6]. Persson et al. also used the breath-hold CT with voluntary deep inspiration [18]. The interfractional variations in the current study were evaluated using end-exhale phase images under free breathing, which has high reproducibility compared with breath-holding.

Several limitations of our study should be mentioned. Firstly, the intrafractional variations evaluated with 4DCT during a few respiration cycles may not be representative of those during treatment in some patients although a single 4DCT is thought to be reliable for the tumour motion in the majority of patients [19]. Therefore, in our institution, we validate margins to compensate for the intrafractional variations, by visual verification with $\mathrm{kV} x$-ray 
$12 / 23$

254 fluoroscopy after the margins are determined based on the simulation 4DCT. Secondly, 255 motion artefacts affected the contouring of tumours and implanted markers evaluated in 256 binned 4DCT images. Because of this uncertainty in contouring, the intrafractional variations 257 for tumours with larger motion may be over- or underestimated [20]. Furthermore, we 258 evaluated the position of markers of $1.5-\mathrm{mm}$ diameter using 4DCT with a $2.5-\mathrm{mm}$ axial slice 259 thickness. The use of 2.5-mm CT slice thickness would affect the accuracy of contouring the 260 markers, with a maximum uncertainty of localising tumours and markers of $1.25 \mathrm{~mm}$ in the SI 261 direction [6]. Those errors could be reduced by acquiring CT images with a thinner axial slice 262 thickness or by the volumetric acquisition [21,22].

\section{Conclusions}

266 Intrafractional variations of the difference between tumour centroid and marker centroid 267 position increased with both tumour motion amplitude and tumour-marker distance. 268 Additionally interfractional variations of the distance between between tumour centroid and 269 marker centroid position were observed up to $2.5 \mathrm{~mm}$. Thus, an appropriate margin to account 270 for these variations should be considered when planning implanted-marker-based beam 271 delivery. 
$13 / 23$

272

273

\section{References}

[1] Blomgren H, Lax I, Naslund I, Svanstrom R. Stereotactic high dose fraction radiation therapy of extracranial tumors using an accelerator. Clinical experience of the first thirty-one patients. Acta Oncol 1995;34:861-70.

[2] Uematsu M, Shioda A, Tahara K et al. Focal, high dose, and fractionated modified stereotactic radiation therapy for lung carcinoma patients: a preliminary experience. Cancer 1998;82:1062-70.

[3] Keall PJ, Mageras GS, Balter JM et al. The management of respiratory motion in radiation oncology report of AAPM Task Group 76. Med Phys 2006;33:3874-900.

[4] Harada T, Shirato H, Ogura S et al. Real-time tumor-tracking radiation therapy for lung carcinoma by the aid of insertion of a gold marker using bronchofiberscopy. Cancer 2002;95:1720-27.

[5] Nelson C, Balter P, Morice RC et al. Evaluation of Tumor Position and PTV Margins Using Image Guidance and Respiratory Gating. Int J Radiat Oncol Biol Phys 2010;76:1578-85.

[6] van der Voort van Zyp NC, Hoogeman MS, van de Water S et al. Stability of markers used for real-time tumor tracking after percutaneous intrapulmonary placement. Int $\mathbf{J}$ Radiat Oncol Biol Phys 2011;81:e75-81.

[7] Seppenwoolde Y, Shirato H, Kitamura K et al. Precise and real-time measurement of 3D tumor motion in lung due to breathing and heartbeat, measured during radiotherapy. Int J Radiat Oncol Biol Phys 2002;53:822-34.

[8] Kothary N, Heit JJ, Louie JD et al. Safety and Efficacy of Percutaneous Fiducial Marker Implantation for Image-guided Radiation Therapy. J Vasc Interv Radiol 2009;20:235-39. 
296 [9] Bhagat N, Fidelman N, Durack JC et al. Complications associated with the percutaneous insertion of fiducial markers in the thorax. Cardiovasc Intervent Radiol 2010;33:1186-91.

[10] Kupelian PA, Forbes A, Willoughby TR et al. Implantation and Stability of Metallic Fiducials Within Pulmonary Lesions. Int J Radiat Oncol Biol Phys 2007;69:777-85.

[11] Imura M, Yamazaki K, Shirato $\mathrm{H}$ et al. Insertion and fixation of fiducial markers for setup and tracking of lung tumors in radiotherapy. Int J Radiat Oncol Biol Phys 2005;63:1442-47.

[12] Schroeder C, Hejal R, Linden PA. Coil spring fiducial markers placed safely using 305 navigation bronchoscopy in inoperable patients allows accurate delivery of CyberKnife stereotactic radiosurgery. J Thorac Cardiovasc Surg 2010;140:1137-42.

[13] Harley DP, Krimsky WS, Sarkar S, Highfield D, Aygun C, Gurses B. Fiducial Marker Placement Using Endobronchial Ultrasound and Navigational Bronchoscopy for Stereotactic Radiosurgery: An Alternative Strategy. Ann Thorac Surg 2010;89:368-74.

[14] Roman NO, Shepherd W, Mukhopadhyay N, Hugo GD, Weiss E. Interfractional positional variability of fiducial markers and primary tumors in locally advanced non-small-cell lung cancer during audiovisual biofeedback radiotherapy. Int J Radiat Oncol Biol Phys 2012;83:1566-72.

[15] Li R, Mok E, Chang DT et al. Intrafraction verification of gated RapidArc by using beam-level kilovoltage X-ray images. Int J Radiat Oncol Biol Phys 2012;83:e709-15.

316 [16] Smith RL, Yang D, Lee A, Mayse ML, Low DA, Parikh PJ. The correlation of tissue motion within the lung: Implications on fiducial based treatments. Med Phys 
319 [17] Yamazaki R, Nishioka S, Date H, Shirato H, Koike T, Nishioka T. Investigation of the change in marker geometry during respiration motion: a preliminary study for dynamic-multi-leaf real-time tumor tracking. Radiat Oncol 2012;7:218.

[18] Persson GF, Josipovic M, Von der Recke P et al. Stability of percutaneously implanted markers for lung stereotactic radiotherapy. J Appl Clin Med Phys 2013;14:187-95.

[19] Guckenberger M, Wilbert J, Meyer J, Baier K, Richter A, Flentje M. Is a single respiratory correlated $4 \mathrm{D}-\mathrm{CT}$ study sufficient for evaluation of breathing motion? Int J Radiat Oncol Biol Phys 2007;67:1352-59.

[20] Persson GF, Nygaard DE, Brink C et al. Deviations in delineated GTV caused by artefacts in 4DCT. Radiother Oncol 2010;96:61-66.

[21] Yamashita H, Kida S, Sakumi A et al. Four-dimensional measurement of the displacement of internal fiducial markers during 320-multislice computed tomography scanning of thoracic esophageal cancer. Int J Radiat Oncol Biol Phys 2011;79:588-95.

[22] Yamashita H, Okuma K, Tada K et al. Four-dimensional measurement of the displacement of internal fiducial and skin markers during 320-multislice computed tomography scanning of breast cancer. Int J Radiat Oncol Biol Phys 2012;84:331-35.

\section{Acknowledgments}

338 This research was supported by the Japan Society for the Promotion of Science (JSPS) 339 through the "Funding Program for World-leading Innovative R\&D on Science and 340 Technology" (FIRST Program).

\section{Conflicts of interest}


$16 / 23$

343 Takashi Mizowaki, Masaki Kokubo, and Masahiro Hiraoka have a consultancy agreement

344 with Mitsubishi Heavy Industries, Ltd., Japan. 
$17 / 23$

\section{Figure legends}

Fig. 1. The range of intrafractional variations in the RL (a), AP (b), and SI (c) directions for each patient rearranged according to the three-dimensional tumour motion amplitude in descending order. Abbreviations: RL, right-left; AP, anterior-posterior; SI, superior-inferior

Fig. 2. $\mathrm{E}_{n}$ values in the RL (a), AP (b), and SI (c) directions for each respiratory phase. $\mathrm{E}_{n}$ is the error in the relative position of the tumour to the centroid of the markers on $\mathrm{n} \%$ phase images $(0 \leq n \leq 90)$, using the relative position on the $50 \%$ phase images as a reference. Other abbreviations are as in Fig. 1.

Fig. 3. Interfractional variation in the RL, AP, and SI directions. Abbreviations are as in Fig.

1.

Table 1. Characteristics of patients and tumours $(n=15)$.

\begin{tabular}{lc}
\hline Characteristics & $n=15$ \\
\hline Age (y) & 82 \\
$\quad$ Median & {$[54-87]$} \\
$\quad$ [range] & \\
\hline Gender & 12 \\
$\quad$ Male & 3 \\
$\quad$ Female & \\
\hline Tumour size & 4 \\
$\leq 20$ mm & 6 \\
$>20$ to $\leq 30$ mm & 5 \\
$>30$ to $\leq 50 \mathrm{~mm}$ & \\
\hline Tumour location & 2 \\
Right middle lobe & 7 \\
Right lower lobe & 2 \\
Left upper lobe & 4 \\
Left lower lobe & 9 \\
\hline No. of implanted markers & \\
4 &
\end{tabular}


$18 / 23$

\begin{tabular}{cc}
5 & 6 \\
\hline No. of markers evaluated & \\
3 & 10 \\
4 & 4 \\
5 & 1 \\
\hline
\end{tabular}


$19 / 23$

Table 2. Predictive factors for the range of intrafractional variation as determined by multiple linear regression analysis.

\begin{tabular}{lccccccc}
\hline & \multicolumn{6}{c}{ Range of intrafractional variation } \\
\cline { 2 - 8 } Predictive factor & \multicolumn{2}{c}{$\mathrm{RL}$} & \multicolumn{3}{c}{ AP } & \multicolumn{3}{c}{ SI } \\
& $\beta$ & $p$ & $\beta$ & $p$ & $\beta$ & $p$ \\
\hline $\begin{array}{l}\text { Tumour motion } \\
\text { amplitude }\end{array}$ & 0.539 & 0.048 & 0.428 & 0.076 & 0.591 & 0.011 \\
Tumour-marker distance & -0.051 & 0.84 & 0.449 & 0.064 & 0.327 & 0.012 \\
$\mathbf{R}$ & \multicolumn{2}{c}{0.549} & \multicolumn{2}{c}{0.649} & \multicolumn{2}{c}{0.780} \\
\hline
\end{tabular}

Abbreviations: R, correlation coefficient; other abbreviations are as in Fig. 1. 
Supplementary Table 1. Summary of reports of interfractional variation between tumours and markers.

\begin{tabular}{|c|c|c|c|c|c|c|}
\hline \multirow{2}{*}{ Author } & \multirow{2}{*}{$n$} & \multirow{2}{*}{ Modality } & \multicolumn{3}{|c|}{ Implanted Markers } & \multirow{2}{*}{ Result } \\
\hline & & & $n$ & Shape & Insertion & \\
\hline $\begin{array}{l}\text { Nelson et al. } \\
\text { [5] }\end{array}$ & 5 & 4DCT & 1 to 4 & Cylinder & Transbronchial & $6 \pm 3 \mathrm{~mm}^{*}$ \\
\hline $\begin{array}{l}\text { Roman et al. } \\
\text { [14] }\end{array}$ & 7 & 4D CBCT & 2 to 4 & $\begin{array}{l}\text { Long } \\
\text { coil }\end{array}$ & Transbronchial & $\begin{array}{l}4 \pm 2 \mathrm{~mm} \text { ( lateral), } \\
3 \pm 2 \mathrm{~mm}(\mathrm{AP}), \text { and } 4 \\
\pm 3 \mathrm{~mm}(\mathrm{SI}) *\end{array}$ \\
\hline $\begin{array}{l}\text { Kupelian et } \\
\text { al. [10] }\end{array}$ & 23 & $\begin{array}{l}\text { Breath-hold } \\
\text { CT (exhale) }\end{array}$ & NA & $\begin{array}{l}\text { Long } \\
\text { coil }\end{array}$ & $\begin{array}{l}\text { Transcutaneous } \\
\text { / Transbronchial }\end{array}$ & $\begin{array}{l}2.6 \pm 1.3 \mathrm{~mm}^{*} \\
\text { (range, } 0.2 \text { to } 5.4 \text { ) }\end{array}$ \\
\hline $\begin{array}{l}\text { Van der } \\
\text { Voort van } \\
\text { Zyp et al. [6] }\end{array}$ & 42 & $\begin{array}{l}\text { Breath-hold } \\
\text { CT (exhale) }\end{array}$ & 3 & Cylinder & Transcutaneous & $\begin{array}{l}\text { Median } 1.3 \mathrm{~mm} \\
\text { (range, } 0.1 \text { to } 53.6 \text { ) }\end{array}$ \\
\hline $\begin{array}{l}\text { Persson et al. } \\
{[18]}\end{array}$ & 14 & $\begin{array}{l}\text { Breath-hold } \\
\text { CT (inhale) }\end{array}$ & 1 & $\begin{array}{l}\text { Long } \\
\text { coil }\end{array}$ & Transcutaneous & $\begin{array}{l}\text { Range: }-2.9 \text { to } 2.6 \\
\mathrm{~mm} \text { (LR), }-1.8 \text { to } 1.5 \\
\mathrm{~mm} \text { (AP), and }-2.6 \text { to } \\
2.8 \mathrm{~mm} \text { (SI) }\end{array}$ \\
\hline This study & 15 & 4DCT $(50 \%)$ & 3 to 5 & Sphere & Transbronchial & $\begin{array}{l}\text { All values in each } \\
\text { direction were within } \\
2.5 \mathrm{~mm}\end{array}$ \\
\hline
\end{tabular}

* mean \pm standard deviation.

Abbreviations: 4D, four-dimensional; CT, computed tomography; CBCT, cone-beam CT; RL, right-left; AP, anterior-posterior; SI, superior-inferior 
(a)
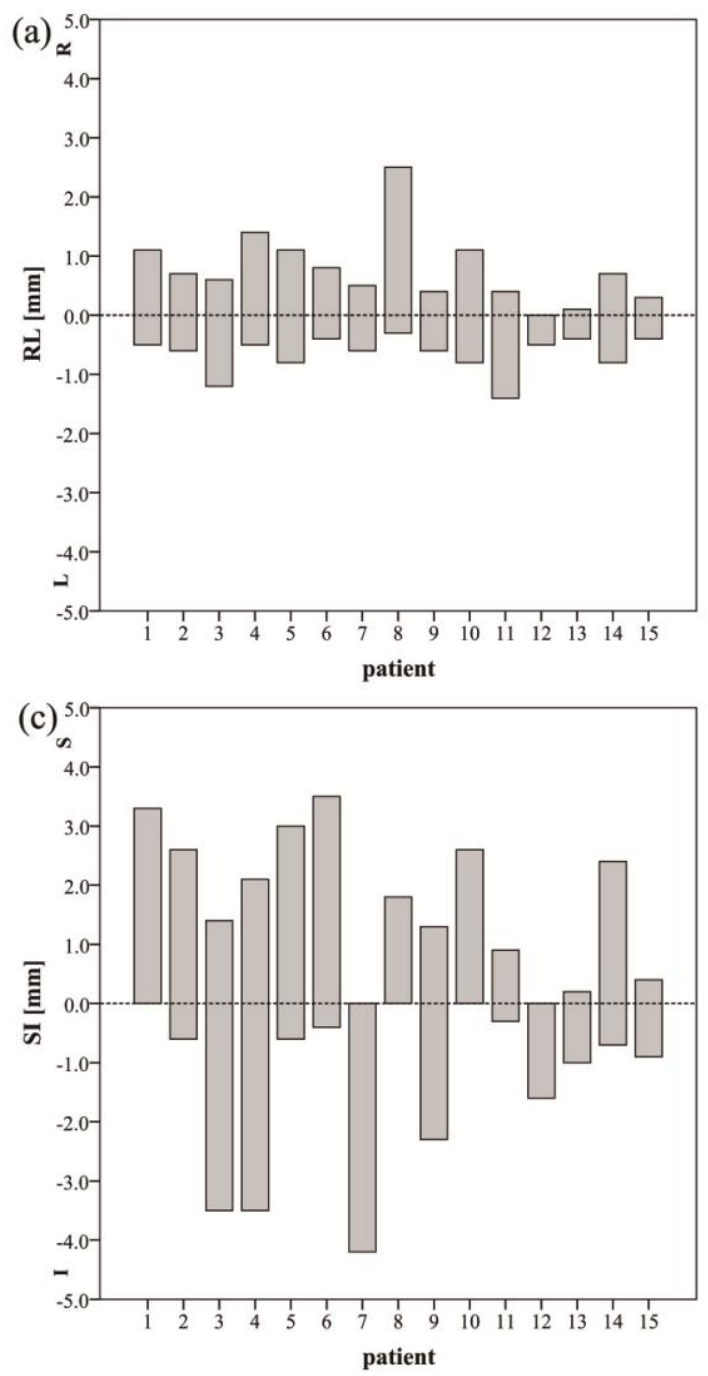

(b)

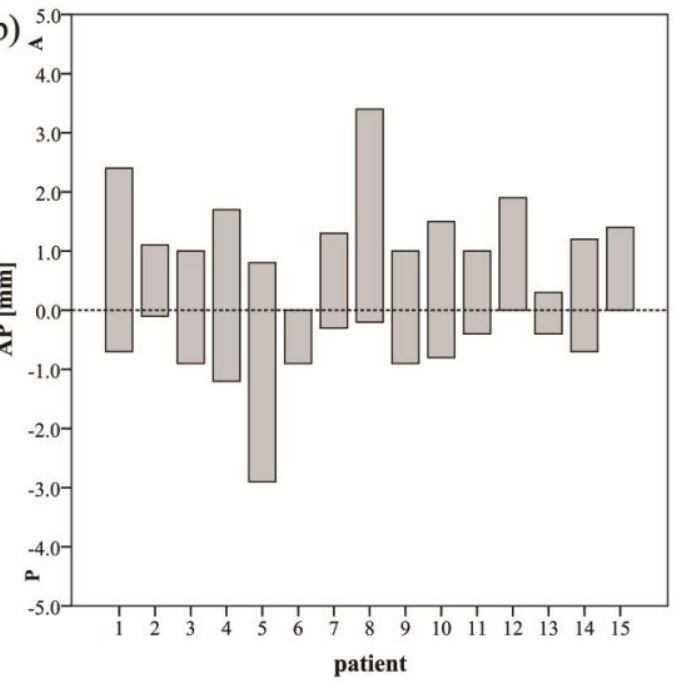

Fig. 1. 

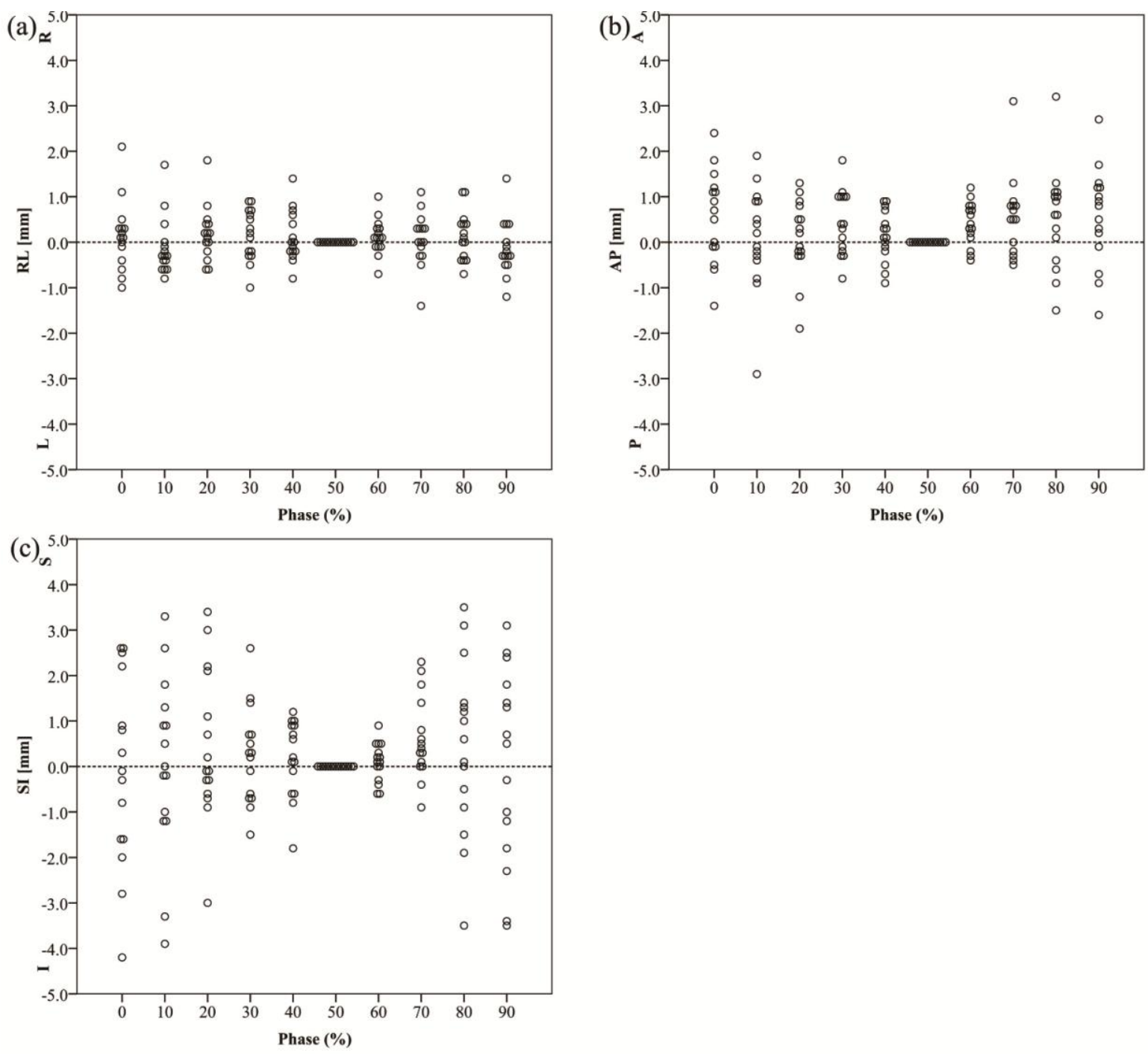

Fig. 2. 


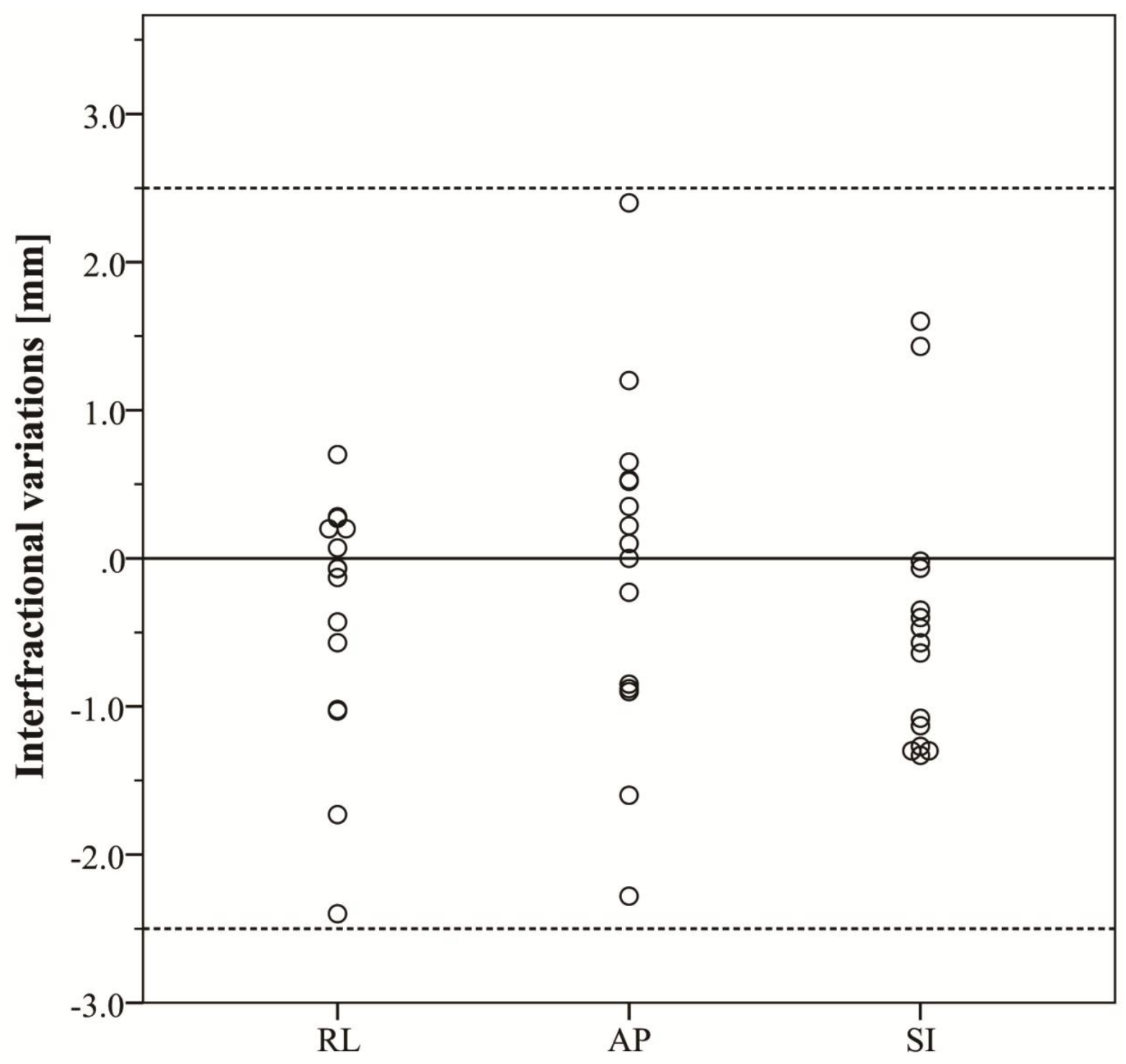

Direction

Fig. 3. 